University of New Hampshire

University of New Hampshire Scholars' Repository

3-1992

\title{
Evaluation and Verification of Bottom Acoustic Reverberation Statistics Predicted by the Point Scattering Model
}

\author{
Dimitri Alexandrou \\ Duke University \\ Christian de Moustier \\ University of California - San Diego \\ G Haralabus \\ Duke University
}

Follow this and additional works at: https://scholars.unh.edu/ccom

Part of the Oceanography and Atmospheric Sciences and Meteorology Commons

\section{Recommended Citation}

D. Alexandrou, 'Evaluation and verification of bottom acoustic reverberation statistics predicted by the point scattering model', The Journal of the Acoustical Society of America, vol. 91, no. 3, p. 1403, 1992.

This Journal Article is brought to you for free and open access by the Center for Coastal and Ocean Mapping at University of New Hampshire Scholars' Repository. It has been accepted for inclusion in Center for Coastal and Ocean Mapping by an authorized administrator of University of New Hampshire Scholars' Repository. For more information, please contact Scholarly.Communication@unh.edu. 


\title{
Evaluation and verification of bottom acoustic reverberation statistics predicted by the point scattering model
}

\author{
D. Alexandrou \\ Department of Electrical Engineering, Duke University, Durham, North Carolina 27706 \\ C. de Moustier \\ Marine Physical Laboratory, Scripps Institution of Oceanography, University of California, San Diego, \\ 9500 Gilman Drive, La Jolla. California 92093-0205
}

G. Haralabus

Department of Electrical Engineering, Duke University, Durham, North Carolina 27706

(Received 26 November 1990; accepted for publication 23 September 1991)

\begin{abstract}
The point scattering model offers a parameterization of the reverberation probability density function (pdf) in terms of the coefficient of excess (kurtosis) and a coherent component represented by a harmonic process with random phase. In this paper the potential utility of this parametrization is investigated in the context of seafloor characterization. The problem of separating out the effect of each parameter is discussed. Computer simulations are used to verify model predictions on the reverberation quadrature, envelope, and phase pdf. As part of the verification study, the scatterer density was determined from the kurtosis of the reverberation quadrature pdf. A statistical analysis of this procedure points to reduced estimate accuracy with decreasing kurtosis. Additional computer simulations show that the chosen pdf family, developed under the assumption of a Poisson scatterer distribution, is flexible enough to fit reverberation data generated by non-Poisson scatterer distributions exhibiting a degree of clustering or regularity. A computer experiment demonstrates how this parametrization can be used in conjunction with a simple sonar geometry to generate acoustic signatures for seafloor classification. In addition, real reverberation data collected by a Sea Beam sonar system in two different seafloor areas are interpreted according to the chosen parametrization.
\end{abstract}

PACS numbers: 43.30.Hw, 43.30.Gv, 43.20.Fn

\section{INTRODUCTION}

Acoustic reverberation is usually modeled as a Gaussian stochastic process. Equivalently, its envelope and phase are assumed to have a Rayleigh and uniform probability density function (pdf), respectively. However, real reverberation data often exhibit deviations from this nominal model. ${ }^{1,2}$ More general and flexible pdf families are then needed to describe the statistical behavior of reverberation. ${ }^{3}$ If reverberation is viewed as interference, incorporating its nonGaussian nature into the design of a detection/estimation processor will lead closer to optimum performance. If reverberation is viewed as an information-bearing signal, deviations from normal statistics convey useful information about the scatterer distributions in the medium and ultimately about the nature of the medium.

In this paper, we adopt the latter viewpoint and concentrate on the problem of seafloor characterization. We focus our attention on a reverberation pdf family supported by the point-scattering model. ${ }^{410}$ This pdf family is parametrized by (1) the coefficient of excess, that is associated with the average number of scatterers contributing to the return and (2) a "coherent component" coefficient. Under certain conditions it is possible to relate these parameters to seafloor characteristics such as rms roughness and correlation area. ${ }^{11}$ However, the point-scattering theory has been developed for random (Poisson) scatterer distributions. Therefore, direct analytical connections to seafloor parameters cannot be obtained when non-Poisson distributions are present. In general, scatterers in the ocean exhibit clustering and/or regularity. To remedy the situation, one approach would be to modify the theory to accommodate such nonPoisson distributions. However, finding mathematical laws to describe the variety of subtle departures from the random distribution encountered in the real ocean is a difficult task. And each time one such law is established, the reverberation theory must be suitably rederived. Here, we take a different approach. Using computer simulations, we establish that the chosen pdf family offers an adequate fit to reverberation data generated by non-Poisson scatterer distributions. Then, we use the obtained parameter fit to generate representative acoustic "feature vectors" that reffect the distinguishing characteristics of each distribution. These feature vectors can in turn be used in conjunction with a pattern recognition methodology to classify the seafloor into one of a known set of seafloor types.

A brief review of the pertinent point scattering theory is given in Sec. I. Particular attention is given to inherent limitations in the joint estimation of the coefficient of excess and the coherent component. In Sec. II the computer simulation 
program REVGEN ( REVerberation GENerator) ${ }^{12,13}$ is used to first simulate the classical point scattering model and then to study reverberation produced by a number of different scatterer distributions deviating from the Poisson distribution. Based on these synthetic data, predictions of the model are validated and a procedure is developed for creating feature vectors representative of the different scatterer distributions. In Sec. III, real acoustic reverberation data collected with a Sea Beam system are interpreted within the model parameters.

\section{THE POINT-SCATTERING MODEL}

The following theory is based on Ol'shevskii's development $^{4,5}$ of the point-scattering model. Reverberation is treated as a random process constructed by a linear superposition of individual echoes emanating from a large number of point reflectors distributed independently in a homogeneous medium. Secondary scattering is assumed negligible. The backscattered signal is described by

$$
x(t)=\sum_{i=1}^{N} a_{i} G\left(t_{i}\right) f\left(t-t_{i}, \epsilon_{\mathbf{i}}\right),
$$

where $f(t)$ is the transmitted signal, $a_{i}$ are the stochastic amplitudes (scatterer acoustic cross sections), $t_{i}$ the stochastic arrival times of the elementary scattered signals, and $N$ is the number of elementary signals contributing to the return at time $t$, itself a stochastic process. The $\epsilon_{1}$ represent other stochastic parameters that may characterize the scatterers (e.g., Doppler). The function $G$ accounts for the sonar's acoustic geometry and gain. For a monostatic sonar,

$$
G\left(t_{i}\right)=g B^{2}\left(\mathbf{r}_{i}\right) v\left(t_{i}\right)
$$

where $g$ is a system gain factor, $B^{2}\left(\mathbf{r}_{i}\right)$ is the two-way beam pattern factor in the direction of the $i$ th scatterer, and $v\left(t_{i}\right)$ represents the two-way propagation loss.

Here we assume that the set $\left\{\epsilon_{i}\right\}$ is empty, so that reverberation is described by the simpler expression

$$
x(t)=\sum_{i=1}^{N} a_{i} G\left(t_{i}\right) f\left(t-t_{i}\right) .
$$

The above can be considered to be a problem in repeated trials. The "experiment" is placing scatterer returns at random in the time interval $(0, T)$, where $T$ is the total time of reception. Let the time interval $t_{a}$ be defined in terms of the transmitted pulse length $\tau$ :

$$
t_{a}=(t-\tau / 2, t+\tau / 2) \text {. }
$$

The returns are assumed to be uniformly distributed throughout the full interval of reception $(0, T)$ where $T \gg t_{a}$. Then, the probability that a single return lies in $t_{a}$ is given by

$$
p_{n}=t_{a} / T
$$

A key assumption of the point scattering model is that $P(N)$, the probability that $N$ scatterer returns arrive during $t_{a}$, is governed by the Poisson law:

$$
P(N)=\left[\left(n_{0} t_{a}\right)^{N} / N !\right] \exp \left(-n_{0} t_{a}\right)
$$

where $n_{0}$ is the average number of scattered signals arriving per unit time.

If the Poisson assumption holds and if the $a_{i}$ and $N$ are statistically independent, it can be shown that the $k$ th-order semi-invariant $\lambda_{k}$ of the process $x(t)$ is given by ${ }^{14}$

$$
\lambda_{k}=n_{0}\left(a^{k}\right) \int_{-\infty}^{+\infty} f^{k}(t) d t,
$$

where $\langle$ ) denotes ensemble averaging.

The $\lambda_{k}$ 's are related to the moments

$$
m_{k}=E\left[x^{k}\right]
$$

and the central moments

$$
\mu_{k}=E\left[\left(x-m_{1}\right)^{k}\right]
$$

of the reverberation process $x(t)$. These moments can be used to obtain an Edgeworth series representation of the probability density function (pdf) of reverberation.

\section{A. Non-Gaussian reverberation: The coefficient of excess}

Following the simplifying assumption of a narrow-band transmitted signal and retaining the first two terms of the Edgeworth series, the normalized reverberation pdf is given by

$$
\begin{aligned}
p\left(x_{n}\right)= & \frac{1}{\sqrt{2 \pi} \sigma} \exp \left(-\frac{x_{n}^{2}}{2}\right) \\
& \times\left[1+\frac{1}{4 !}\left(\frac{\mu_{4}}{\sigma^{4}}-3\right) H_{4}\left(x_{n}\right)\right],
\end{aligned}
$$

where $x_{n}=x / \sigma$ and $H_{4}\left(x_{n}\right)$ is the fourth order Hermite polynomial. Here, $\sigma^{2}$ is the variance of the process $x(t)$. Equivalently

$$
\begin{aligned}
p\left(x_{n}\right)= & \frac{1}{\sqrt{2 \pi} \sigma} \exp \left(-\frac{x_{n}^{2}}{2}\right) \\
& \times\left(1+\frac{\gamma}{4 !}\left(x_{n}^{4}-6 x_{n}^{2}+3\right)\right)
\end{aligned}
$$

where

$$
\gamma=\left(\mu_{4} / \sigma^{4}-3\right)
$$

is known as the coefficient of excess or kurtosis. It is a nondimensional quantity that describes the peakness or flatness of the pdf relative to a Gaussian. As the number of scatterers becomes infinite, $\gamma$ tends to zero and $p\left(x_{n}\right)$ converges to a Gaussian pdf

The coefficient of excess can be related to $\langle N\rangle$, the average number of scatterers combining to produce the reverberation process, via

$$
\langle N\rangle=\frac{\left\langle a^{4}\right\rangle \int_{-\infty}^{\infty} f^{4}(t) d t}{\gamma\left\langle a^{2}\right\rangle^{2}\left[\int_{-\infty}^{\infty} f^{2}(t) d t\right]^{2}} .
$$

For a rectangular transmitted pulse $f(t)$ and a Rayleighdistributed scatterer amplitude $a$ the above reduces to

$$
\langle N\rangle=3 / \gamma
$$

The practical utility of this expression will be investigated in Sec. II.

The complex envelope of reverberation $\chi(t)$ is given by

$$
\chi(t)=x_{c}(t)+j x_{s}(t)
$$

where $x_{c}(t)$ and $x_{s}(t)$ are the reverberation quadrature components, each distributed according to Eq. (11). The 
quantity usually referred to as the reverberation "envelope" is

$$
u(t)=|\chi(t)|=\sqrt{x_{\mathrm{c}}^{2}(t)+x_{s}^{2}(t)} .
$$

The pdf of the normalized reverberation envelope is given by

$$
\begin{aligned}
p\left(u_{n}\right)= & \frac{u_{n}}{\sigma} \exp \left(-\frac{u_{n}^{2}}{2}\right)\left[1+\frac{\gamma}{4 !}\left(\frac{3}{4} u_{n}^{4}-6 u_{n}^{2}+6\right)\right. \\
& +\left(\frac{\gamma}{4 !}\right)^{2}\left(\frac{3}{128} u_{n}^{8}-\frac{3}{4} u_{n}^{6}\right. \\
& \left.\left.+\frac{27}{4} u_{n}^{4}-18 u_{n}^{2}+9\right)\right],
\end{aligned}
$$

where $u_{n}=u / \sigma$. As $\gamma$ tends to zero, $p\left(u_{n}\right)$ converges to a Rayleigh pdf.

\section{B. Influence of a coherent component}

A potentially useful extension of the pdf family defined by Eq. (11) is in terms of a coherent (quasideterministic) component embedded in the reverberation return. The point-scattering model offers two such generalizations. The first involves a constant term $E_{0}$ added to the scattered return. In this case, the reverberation pdf becomes

$$
\begin{aligned}
p\left(x_{n}\right)= & \frac{1}{\sqrt{2 \pi} \sigma} \exp \left(-\frac{\left(x_{n}-Q\right)^{2}}{2}\right) \\
& \times\left(1+\frac{\gamma}{4 !}\left[\left(x_{n}-Q\right)^{4}-6\left(x_{n}-Q\right)^{2}+3\right]\right),
\end{aligned}
$$

where $Q$ is the normalized coherent component coefficient defined by

$$
Q=E_{0} / \sigma
$$

The type of coherent component implied by Eq. (18) is physically meaningful when a large and constant signal combines with reverberation. For instance, a specular component embedded in reverberation at near-normal incidence could give rise to this "single glint" scenario. The corresponding envelope pdf ${ }^{5}$ is an extension of the classical Ricean pdf.

According to the second and more interesting generalization, the coherent component is represented by a harmonic process with random phase. In this case, the composite reverberation return is given by

$$
x(t)=x_{r}(t)+x_{0},
$$

where $x_{r}$ is the reverberation component governed by the pdf of Eq. (11) and $x_{0}$ is the coherent component given by

$$
x_{0}=E_{0} \cos \theta,
$$

where $\theta$ is uniformly distributed in $(-\pi, \pi)$. The pdf of $x_{0}$ is well known and given by

$$
p\left(x_{0}\right)=1 / \pi\left(E_{0}^{2}-x_{0}^{2}\right)^{1 / 2} .
$$

The pdf of the composite reverberation return is obtained by convolving the above with Eq. (11). The result is

$$
\begin{aligned}
p\left(x_{n}\right)= & \frac{1}{\pi \sqrt{2 \pi} \sigma} \int_{0}^{\pi} \exp \left(-\frac{\left(x_{n}-Q \cos \theta\right)^{2}}{2}\right) \\
& \times\left(1+\frac{\gamma}{4 !}\left[\left(x_{n}-Q \cos \theta\right)^{4}\right.\right. \\
& \left.\left.-6\left(x_{n}-Q \cos \theta\right)^{2}+3\right]\right) d \theta
\end{aligned}
$$

where, once again, $x_{n}=x / \sigma$ and $Q=E_{0} / \sigma$. The coherent component expressed by Eq. (23) is particularly interesting because it can potentially represent a number of realistic scattering scenarios. For example, a moving sonar platform may result in a fluctuating specular component received by a downlooking beam. Alternatively, occasional large scatterers of varying size or intermittent coherent scattering from ordered scatterer distributions may result in a composite process of this type. Because this formulation is more powerful and to the best of our knowledge has not been explored elsewhere, it is the focus of our interest here.

No corresponding envelope pdf expression is available for the second type of quasideterministic component (random harmonic process). For this reason, we consider it preferable to work with the reverberation quadrature components [Eqs. (10) and (23)] rather than the reverberation envelope. In the case of $\mathrm{Eq}$. (23) we have used numerical integration to obtain an approximation of the desired pdf expression.

\section{Simultaneous estimation of $\gamma$ and $Q$}

Representative curves for the pdf of Eq. (23) are shown in Fig. 1. The effect of increasing $Q$ is seen to be a flattening of the pdf relative to a Gaussian. That is, a strong coherent component of this type leads to negative excess (kurtosis). This immediately points to a problem in estimating $Q$. Specifically, it is not possible to estimate $\gamma$ independently of the influence of $Q$ based on the pdf of the received process. One would need a priori information about the "true" $\gamma$ in the absence of a coherent component.

An additional problem arises from the presence of the quantity $\sigma$, which is the standard deviation of the pure seattered component of the received process and not of the entire received process, including the coherent component. Thus, unless sufficient information exists about the reverberation process devoid of the coherent component, it is not possible to properly normalize the pdf estimate.

Therefore, in general, it is not possible to isolate the $\gamma$ and $Q$ of a composite reverberation process. If the true $\gamma$ is known, then it should be possible to estimate $Q$ with the assistance of a goodness-of-fit test. If one attempts to estimate $\gamma$ directly from the data, one would actually estimate an "effective" coefficient of excess $\gamma_{\text {eff }}$, which potentially includes the influence of a coherent component. If this $\gamma_{\text {eff }}$ is accepted as the true $\gamma$, the pdf fitting procedure will greatly underestimate the value of $Q$.

Monte Carlo simulations were used to illustrate this point. Random variables were created governed by the pdf of (23) with a range of (true) $\gamma$ and $Q$ values. The $\gamma_{\text {eff }}$ was estimated using the moment method (see Sec. II B). The results are shown in Fig. 2: As $Q$ increases, $\gamma_{\text {eff }}$ is increasing- 

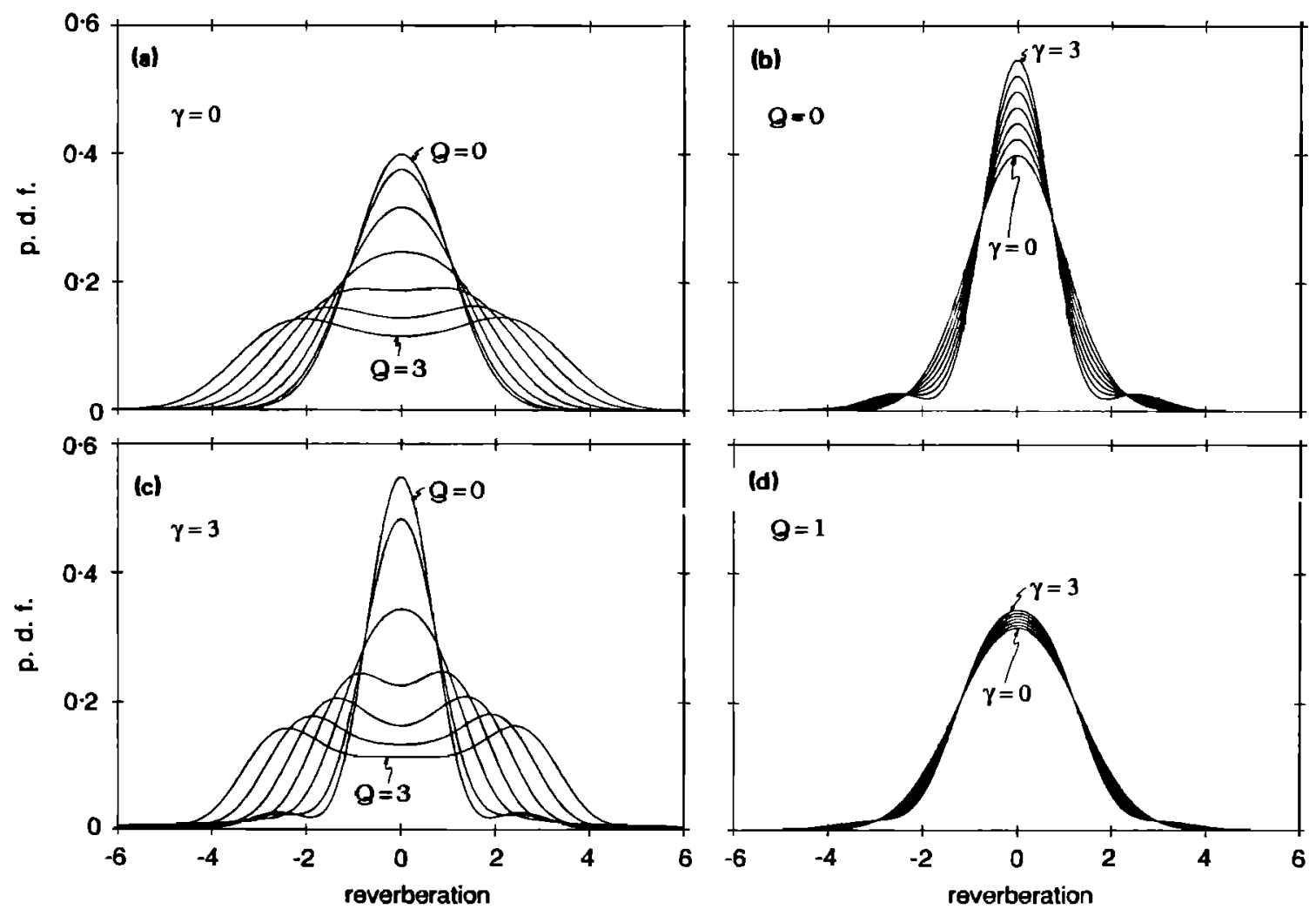

FIG. 1. Examples of the pdf of Eq. (23) illustrating the influence of varying $\gamma$ and $Q$. In each case, the variable quantity varies from $0-3$ with an increment of 0.5 .

ly smaller than the true $\gamma$ and eventually reaches negative values.

In practice, one would have to be content with using $\gamma_{\text {eff }}$ alone to characterize the underlying scatterer distribution in terms of both the effective number of scatterers and the presence/absence of a coherent component. Because of the diffi-

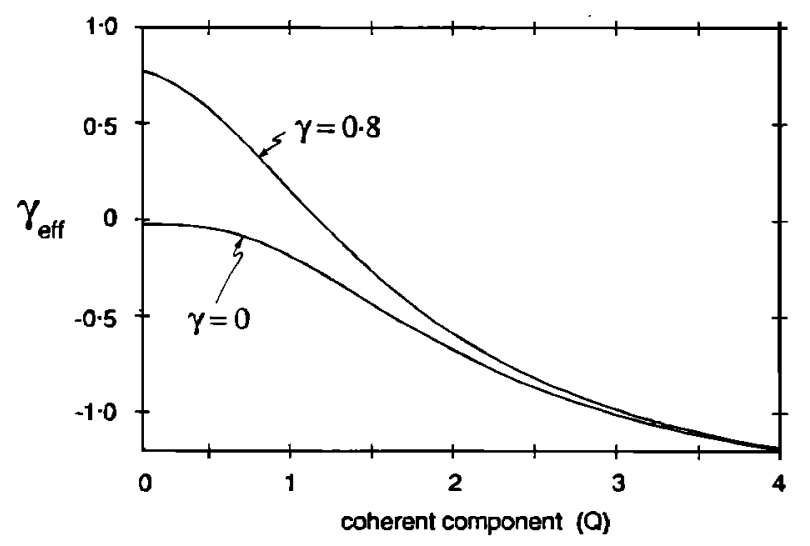

FIG. 2. The influence of a coherent component on the estimate of the coefficient of excess: As $Q$ increases, $\gamma_{\text {eff }}$ is increasingly smaller than the true $\gamma$ and eventually becomes negative. culties described above, it will not be possible to quantify these two effects separately. However, this formulation may still be useful when considered in conjunction with additional information that may be available in a particular experimental setting. For example, under certain conditions, it may be reasonable to assume that a sufficiently large number of scatterers exists so that $\gamma \sim 0$. If $\gamma_{\text {eff }}$ is found to be negative, this would be a strong indication that a coherent component is present in the return. The underlying value of $Q$ may then be obtained with the assistance of a "calibration" curve of the type shown in Fig. 2. As an alternative, in the limit $\gamma=0$, finding $Q$ can be considered a problem in optimum parameter estimation in the presence of Gaussian noise. This may be a viable approach and one deserving further consideration.

\section{COMPUTER SIMULATIONS}

The simulation program REVGEN (REVerberation GENerator) amounts to a direct implementation of the point scattering model. It creates random distributions of scatterers, it insonifies them with with a specified waveform, and yields the reverberation quadrature components. REVGEN is very flexible in representing system characteristics and is a welltested environment that has been utilized in a number of applications. ${ }^{15-17}$ In this section, REVGEN simulations involving realistic sonar geometries and signaling are used to investigate the potential of the chosen pdf parametrization in the context of seafloor classification. 


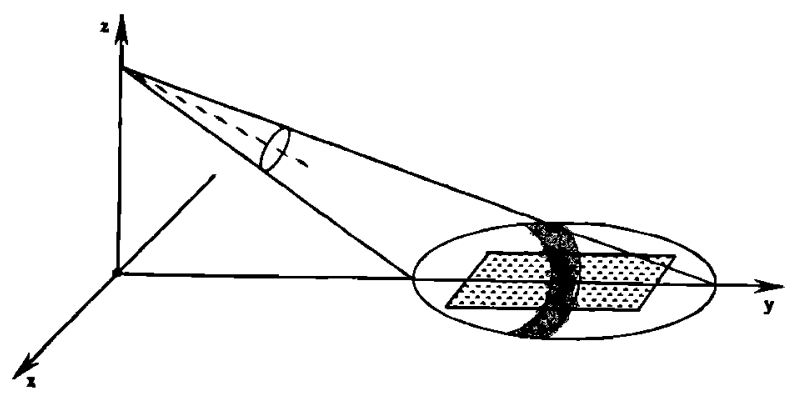

FIG. 3. Simulation geometry.

\section{A. Model verification}

Before proceeding with the main objective, it is deemed necessary to begin with a verification experiment. The reaSon is that REVGEN normally creates Poisson scatterer distributions and allows the density and amplitude distribution of the scatterers to vary with range. This is done for the sake of efficiency, because increasingly numerous scatterers are needed to represent the increasing insonified area with increasing range. REVGEN inserts fewer but larger scatterers as the range increases. This could disturb the homogeneity of the distribution, creating departures from the model predictions. Therefore, scatterer distributions were created externally to REVGEN and inserted by utilizing the program option to accept arbitrarily located individual scatterers. This allows the strict control of the scatterer populations and makes possible the creation of different types of distributions. On the other hand, this unorthodox way of using REVGEN requires careful testing because it bypasses normal program controls and is not bound by past model verification studies.

The geometry of the verification simulation is shown in Fig. 3. The scatterers are distributed within a square measuring $100 \mathrm{~m}$ on a side. They are ideal point reflectors with a Poisson spatial distribution and Rayleigh-distributed amplitudes. The source is placed $20 \mathrm{~m}$ off the bottom at a horizontal distance of $300 \mathrm{~m}$ away from the scatterers. The transmitted signal is a rectangular pulse with a carrier frequency of 1 MHz. The pulse length is set to $10 \mathrm{~ms}$; this is sufficiently long to assure that enough scatterers are contributing to the return at any instant to create a Gaussian reverberation process. The beam patterns are simple cones, the sound speed profile is constant with depth, and the ocean is perfectly noiseless. The only signal received is the collective contribution of the scatterers. A number of pings were transmitted. Each time, the reverberation return was sampled at the $\mathrm{Ny}$ -
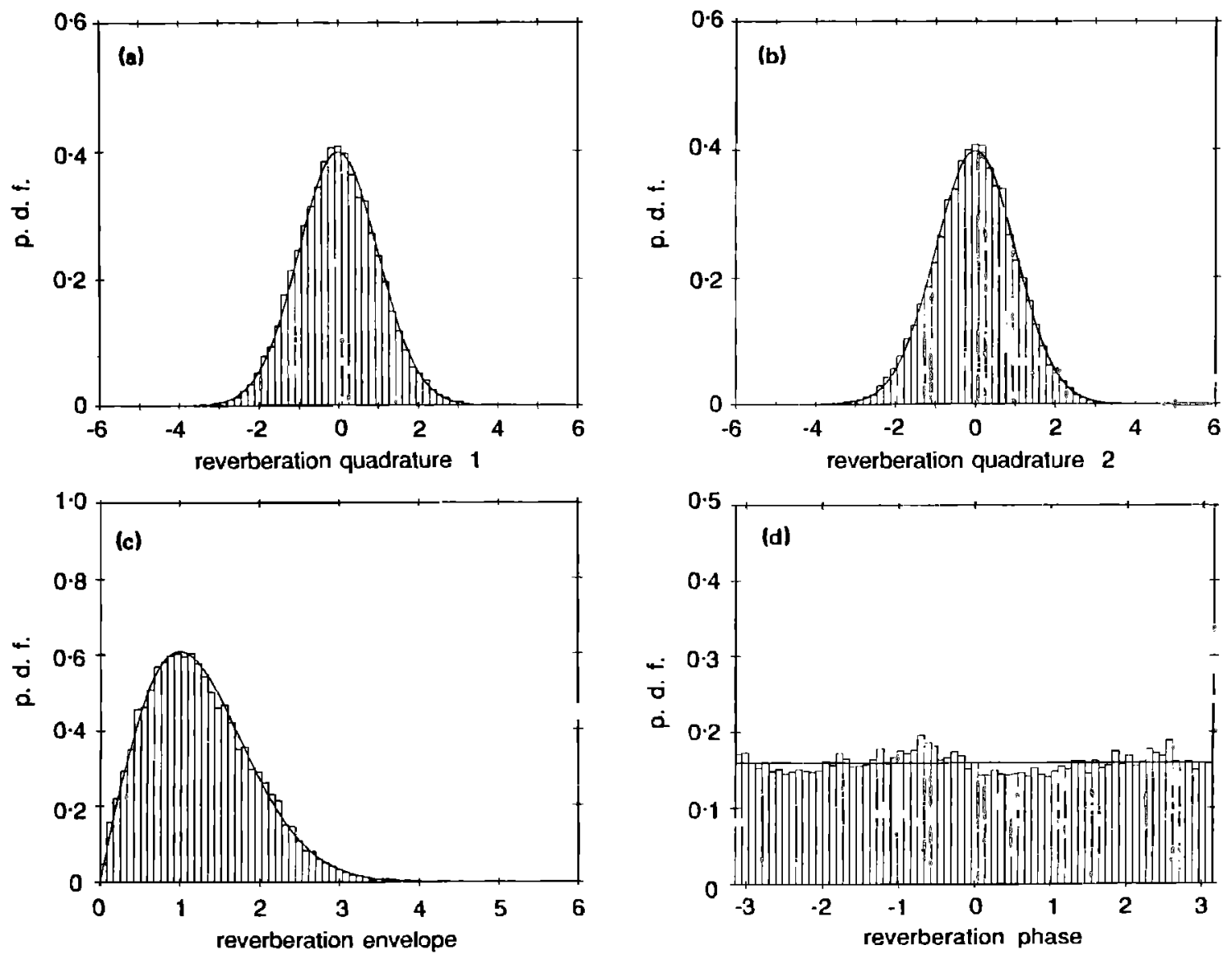

FIG. 4. Results of the verification experiment: (a). (b) The quadrature components are Gaussian, (c) the envelope is Rayleigh, (d) and the phase is uniformly distributed. 
quist rate. Care was taken to avoid edge effects associated with the initial onset and the departure of the pulse from the scattering area. Following corrections for transmission loss due to spherical spreading (the model does not include absorption), returns from consecutive pings were concatenated to form a time series 30000 samples long. This record, normalized to unit variance, forms the basis for our statistical estimates.

The expected result is Gaussian-distributed reverberation quadrature components, Rayleigh-distributed reverberation envelope, and uniformly distributed phase. Equivalently, $\gamma$ must approach zero. Reverberation quadrature, envelope, and phase histograms obtained from the simulated data are shown in Fig. 4. They closely resemble the predicted outcome. In order to make a quantitative statement, Kolmogorov's goodness-of-fit test ${ }^{18}$ was used to determine the match between the theoretical and experimental pdfs. The quadrature components passed the null hypothesis (Gaussian distribution) at a confidence level of $95 \%$. Similarly, the envelope and phase passed the null hypothesis (Rayleigh and uniform, respectively) at a confidence level of $95 \%$.

\section{B. Experimental determination of scatterer density}

As shown in Sec. I A, the coefficient of excess $\gamma$ is related to the number of scatterers contributing to the return
[Eq. (14)]. When $\gamma$ is not equal to zero, this expression yields an estimate of the scatterer density. Here, this expression is applied to recover $\langle N\rangle$ from estimates of $\gamma$ obtained from synthetic reverberation data. This may be viewed as part of the verification study since the number of scatterers inserted in the simulation is exactly known $a$ priori. In addition, the practical utility of this model prediction can be evaluated in light of the parameter estimation problem involved.

The coefficient of excess was estimated according to the classical moment method as follows:

$$
\hat{\gamma}=M_{4} / M_{2}^{2}-3
$$

where the sample moments $M_{k}$ are computed directly from $n$ reverberation samples by

$$
M_{k}=\frac{1}{n} \sum_{i=1}^{n}\left(x_{i}-\hat{x}\right)^{k}
$$

and $\hat{x}$ is the sample mean:

$$
\hat{x}=\frac{1}{n} \sum_{i=1}^{n} x_{i}
$$

The variance of $\hat{\gamma}$ denoted here by $s^{2}(\hat{\gamma})$ is given approximately by ${ }^{19}$

$$
s^{2}(\hat{\gamma})=\frac{\mu_{2}^{2} \mu_{6}-4 \mu_{2} \mu_{4} \mu_{6}-8 \mu_{2}^{2} \mu_{3} \mu_{5}+4 \mu_{4}^{3}-\mu_{2}^{2} \mu_{4}^{2}+16 \mu_{2} \mu_{3}^{2} \mu_{4}+16 \mu_{2}^{3} \mu_{3}^{2}}{\mu_{2}^{6} n}
$$

When the parent population is normal, this reduces to

$$
s^{2}(\hat{\gamma})=24 / n \text {. }
$$

In our case, normality cannot be assumed. In fact, this computer experiment is designed to cause a gradual departure from normality toward nonzero values of $\gamma$. Therefore, it is necessary to estimate the variance of $\hat{\gamma}$ as a function of the true value $\gamma$. The instantaneous reverberation pdf including the influence of $\gamma$ is given by Eq. (11). Using this pdf the required central moments were computed by numerical integration and $s^{2}(\hat{\gamma})$ was estimated as a function of $\gamma$ according to Eq. (27). The estimates obtained for selected values of the true value $\gamma$ are shown in Table I. The variance of the estimate, $\hat{\gamma}$, increases as the true value of $\gamma$ increases. However, when estimating $\langle N\rangle$ from $\hat{\gamma}$, the variance of the latter becomes increasingly significant with decreasing $\hat{\gamma}$. This is expressed by the normalized standard error or coefficient of variation $\epsilon_{r}$ :

$$
\epsilon_{r}=s(\hat{\gamma}) / \hat{\gamma}
$$

Thus, the quality of the $\langle N\rangle$ estimate is expected to deteriorate with decreasing $\hat{\gamma}$. Values listed in Table I indicate that the decrease of $s(\hat{\gamma})$ with $\hat{\gamma}$ is not fast enough to reverse this trend, but only slows it down somewhat.

A REVGEN simulation was used to evaluate the potential of this method for determining scatterer density. During this computer experiment, a progressively shorter pulse is trans- mitted. In each case, $\hat{\gamma}$ is estimated from 30000 reverberation samples. As the pulse length decreases, a progressively smaller area is insonified and correspondingly a smaller number of point scatterers contributes to the return. This should result in a departure from Gaussian instantaneous reverberation statistics expressed by an increase in the kurtosis $\hat{\gamma}$. As $\hat{\gamma}$ becomes nonzero, an estimate of $\langle N\rangle$ becomes possible according to Eq. (14). The quality of this estimate

TABLE I. Theoretical predictions of the variance of $\hat{\gamma}$ when the estimate is made with the method of sample moments. Note that the variance increases with the true value of $\gamma$.

\begin{tabular}{cc}
\hline \hline$\gamma$ & $s^{2}(\hat{\gamma})$ \\
\hline 0.0 & $23.989 / n$ \\
0.2 & $37.387 / n$ \\
0.4 & $48.978 / n$ \\
0.6 & $58.956 / n$ \\
0.8 & $67.512 / n$ \\
1.0 & $74.838 / n$ \\
1.2 & $81.125 / n$ \\
1.4 & $86.565 / n$ \\
1.6 & $91.351 / n$ \\
1.8 & $95.674 / n$ \\
2.0 & $99.726 / n$ \\
\hline
\end{tabular}


will be poor for the longer pulses, where the standard deviation $s(\hat{\gamma})$ is of the same order of magnitude as the true value of $\gamma$. The estimate should improve as the pulses become shorter and $\gamma$ increases. This rationale is well supported by the results. The true value of $\langle N\rangle$ as a function of pulse length and the estimate $\langle\hat{N}\rangle$ obtained with this method are shown in Table II. Both $\langle N\rangle$ and $\langle\hat{N}\rangle$ are rounded off to the nearest integer. Excellent agreement is obtained for pulse lengths shorter than $1.3 \mathrm{~ms}$. For longer pulses the variance of $\hat{\gamma}$ causes significant errors.

\section{Feature vectors in terms of $\gamma$}

At this point it is clear that the simulations are successful in implementing the point scattering model. It is now safe to proceed with the study of alternative scatterer distributions. The Poisson assumption employed by the point-scatterer model restricts the type of physical scattering surfaces that can be treated through this approach. First, the independence assumption is likely to be violated. It is often the case that the physical size of individual scatterers impacts on the placement of their neighbors. In addition, the assumption of uniform scatterer distributions throughout the reception interval is too restrictive. Realistic scatterer distributions typically exhibit a degree of "patchiness" or clustering. Perhaps the most applicable physical scenario is that of manganese nodule distributions.

It seems reasonable to expect the differences in the discrete spatial distribution of the scatterers to be reflected in the statistical behavior of the reverberation produced by these distributions. The result may be acoustic "feature vectors" that could serve as useful markers of different seaffoor provinces. REVGEN simulations give us the opportunity to investigate this possibility.

Three classes of alternative point distributions were created, each departing from the standard Poisson distribution in a different manner. One is a cluster process whereby "parent" points are placed randomly with a certain Poisson density and a random number of "descendant" points are distributed randomly around each parent point, but with a different Poisson density. Two cluster distributions were created, differing in the density of the descendant points.

TABLE II. Computer simulation results: The estimate $\hat{\gamma}$ and the corresponding estimate of the number of scatterers contributing to the return $(\langle\hat{N}\rangle)$ are given for each pulse length $\tau$. Note that $\langle\hat{N}\rangle$ is a good approximation of the true $(N)$ for $\tau<1.3$. For longer pulses, the two values diverge.

\begin{tabular}{ccrr}
\hline \hline$\tau(\mathrm{ms})$ & $\hat{\gamma}$ & $\langle\hat{N}\rangle$ & $\langle N\rangle$ \\
\hline 0.1 & 1.210 & 3 & 2 \\
0.4 & 0.330 & 9 & 6 \\
0.8 & 0.204 & 15 & 13 \\
1.0 & 0.178 & 16 & 14 \\
1.1 & 0.172 & 17 & 15 \\
1.3 & 0.144 & 20 & 19 \\
2.5 & 0.027 & 110 & 37 \\
5.0 & 0.105 & 30 & 73 \\
10.0 & 0.090 & 33 & 140 \\
\hline \hline
\end{tabular}

One (C1) has highly concentrated clusters and the other (C2) fairly diffuse ones. The second class of non-Poisson distribution is a regular or ordered process. In this case, each point is drawn from a Poisson distribution, but is assigned a circular region of exclusion where no other point can be located. Two ordered distributions were created differing in the size of the region of exclusion. This region has a radius of $1 \mathrm{~m}$ for the first distribution (OI) and $1.5 \mathrm{~m}$ for the second $(\mathrm{O} 2)$. The third type of distribution (P2) is a composite of two independent Poisson distributions. Including the simple Poisson (P1), a total of six point distributions were created (Fig. 5).

The sonar geometry and signaling of the verification simulation are used here as well. The variable pulse length allows the interrogation of different spatial scales within each distribution thus allowing the different patterns of scatterer distribution to be manifested in the statistical character of the reverberation process.

For each distribution and pulse length, the coefficient of excess $\gamma$ was estimated using the moment method. Once again, the data set consisted of a total of 30000 samples created in a manner similar to the control experiment. The coefficient of excess $\hat{\gamma}$ was estimated for each type of distribution and is shown as a function of pulse length in Fig. 6 . The $\hat{\gamma}$ signature differs significantly among the three distribution types. In the case of $P 1$ it decreases approximately linearly with increasing pulse length, as expected. Distribution $\mathbf{P} 2$ is initially dominated by the sparse distribution of large scatterers; this leads to large $\hat{\gamma}$ values that fall precipitously with increasing pulse length. For the longer pulses it behaves similarly to P1. Distributions $\mathbf{C 1}$ and $\mathrm{C} 2$ display generally high values of $\hat{\gamma}$ that remain fairly constant after an initial small drop. Here $\mathrm{C} 1$ results in higher $\hat{\gamma}$ values as expected. The $\hat{\gamma}$ values attained by the ordered distributions (O1 and $\mathrm{O} 2$ ) are relatively small and remarkably independent of pulse length for the longer pulses.

These initial results indicate that $\hat{\gamma}$ may be useful as a feature vector for seafloor classification. ${ }^{20}$ More diverse and unique feature vectors can be obtained with more complex sonar geometry and/or signaling.

\section{SEA BEAM DATA ANALYSIS}

In this section, samples of seafloor acoustic backscatter collected with the Sea Beam multibeam echo sounder are used to assess whether real reverberation data can be meaningfully interpreted within the chosen pdf parametrization. The Sea Beam system combines a hull-mounted narrow beam echo sounder with an echo processor to produce on line a high-resolution contour map of a swath of seafloor, roughly three-fourths of the water depth in width, for each traverse of the ship. The echo sounder uses a cross-fanedbeam geometry, whereby a 7-ms continuous-wave pulse of $12.158 \mathrm{kHz}$ is transmitted on a beam $22 / 3$ by $54^{\circ}$, and bottom echoes are received on 16 electronically steered beams each $20^{\circ}$ by $2^{\circ} 2 / 3$ and spaced $2^{\circ} 2 / 3$ apart athwartships (Fig. 7). The echo processor detects and digitizes the echoes received on the 16 beams and performs time and angle of arrival computations to determine depths and horizontal distances on each beam. These bathymetric data are then 

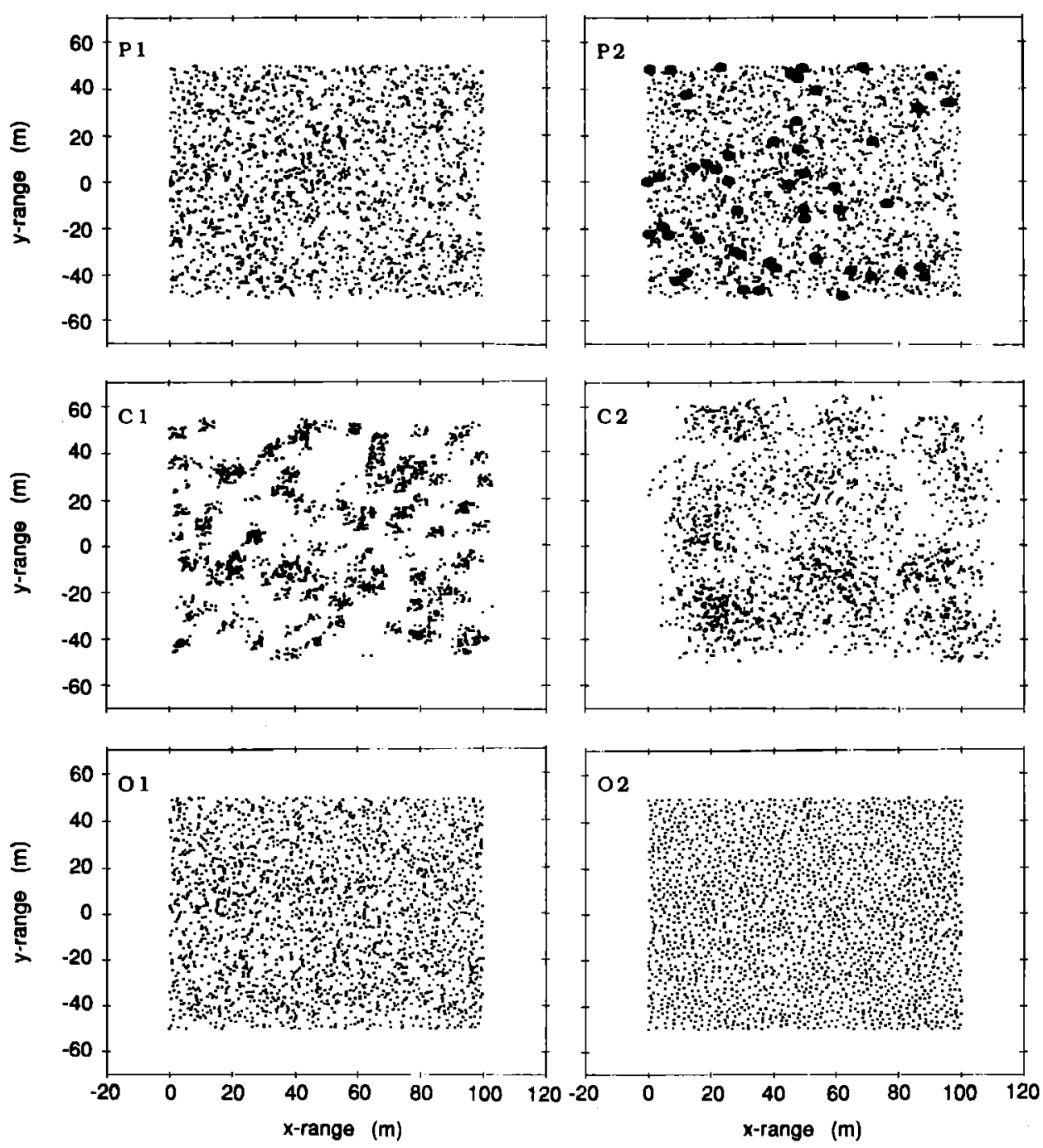

FIG. 5. Examples of two-dimensional point-scatterer distributions: Poisson (P1, $\mathrm{P} 2)$, cluster $(\mathrm{Cl}, \mathrm{C} 2)$, and ordered ( $\mathrm{Ol}$, O2). then displayed in near-real time as a contour map of the swath of seafloor surveyed. Detailed discussions of the Sea Beam system are available elsewhere. ${ }^{21-23}$

Although this system is primarily a bottom mapping tool, its multi-narrow-beam configuration is also well suited for studying the acoustic backscattering characteristics of the seafloor. Previous work with the envelopes of acoustic bottom returns received by the Sea Beam system, has shown that useful information can be gained about seafloor roughness. ${ }^{24}$ However, for this application, the quadrature components of the backscatter process are needed. To this end, data were recorded with a special purpose complex acoustic data acquisition system, ${ }^{25}$ which preserves the quadrature components of the echoes received on each of the 16 preformed beams, along with the ship's roll. These data were processed to remove sidelobe interferences, to correct for geometric and attitude effects (ship's roll, raybending, and bottom slopes), for system related effects (beam patterns, pulse length versus area insonified) and for transmission losses through the water column. These correction procedures are described in detail elsewhere ${ }^{26}$ and will not be repeated here.
The resulting set of quadrature components are placed into angular bins $2^{\circ}$ wide, and statistical analysis is performed within each angular bin. Two data sets recorded over the summit of seamounts in the central North Pacific were subjected to this type of analysis. The first set was recorded in $1500 \mathrm{~m}$ of water over Horizon Guyot, and the second set over Magellan Rise at $3100 \mathrm{~m}$ depth. Both sites are covered with sediments over $150 \mathrm{~m}$ thick.

Estimates of $\gamma$ were made for each angular bin. Plots of $\hat{\gamma}$ as a function of beam angle are shown in Figs. 8 and 9. As previously stated, this $\hat{\gamma}$ is an "effective" kurtosis $\gamma_{\text {eff }}$, which may include the influence of a coherent component. The authors wish to acknowledge that, in view of the limited information available about the two seafloor areas, the following interpretation is necessarily somewhat speculative and is not suggested as the only possible explanation of the observed data behavior.

The Magellan Rise data set (Fig. 8) exhibits the following behavior: In the outer beams, $\gamma_{\text {eff }}$ is effectively zero, implying that a large number of scatterers contributes to the return. There is little evidence of a coherent component. As the angle bins get closer to vertical incidence, $\gamma_{\text {eff }}$ increases 

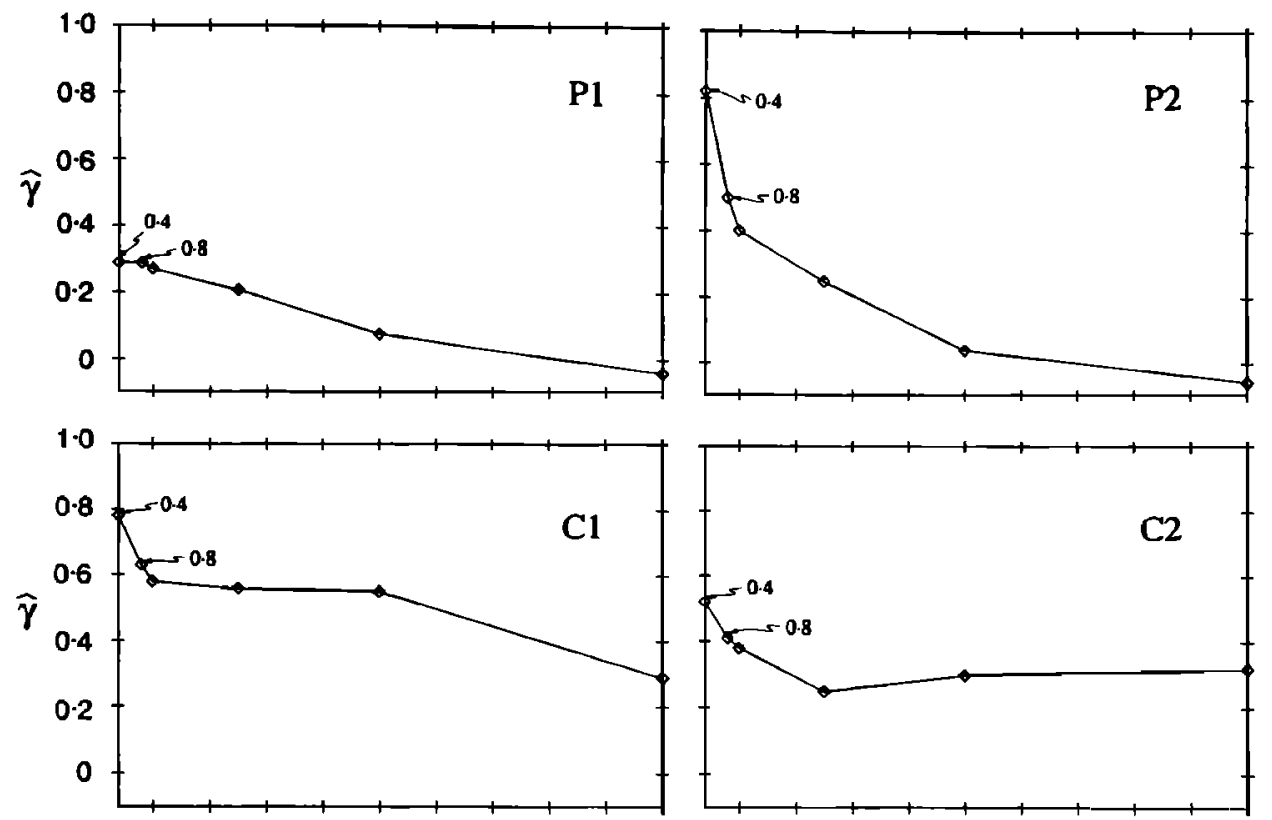

FIG. 6. The coefficient of excess $\hat{\gamma}$ as a function of transmitted pulse length for point distributions $\mathrm{Pl}, \mathrm{P2}, \mathrm{Cl}, \mathrm{C} 2, \mathrm{Ol}$, and $\mathrm{O} 2$.
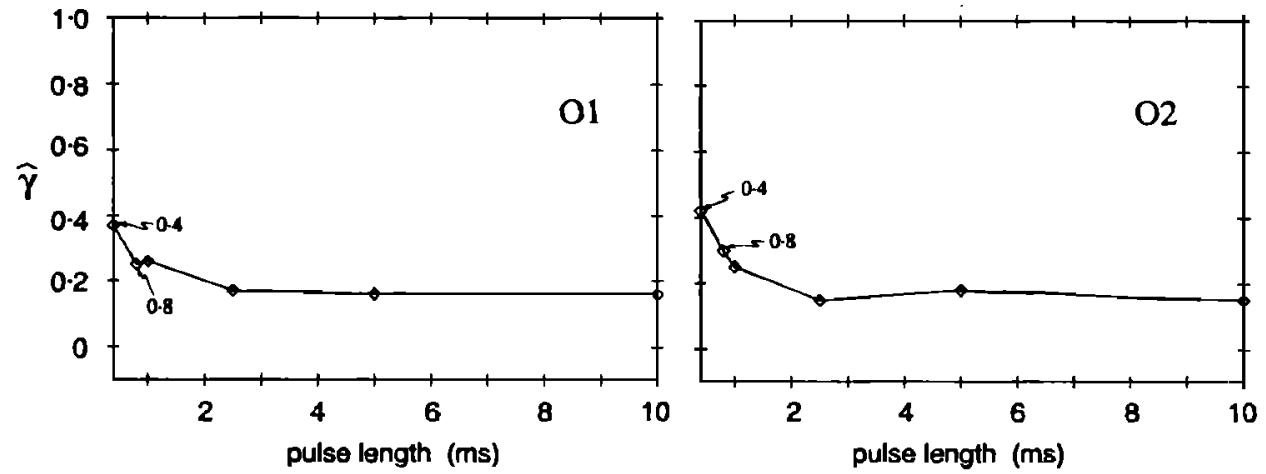

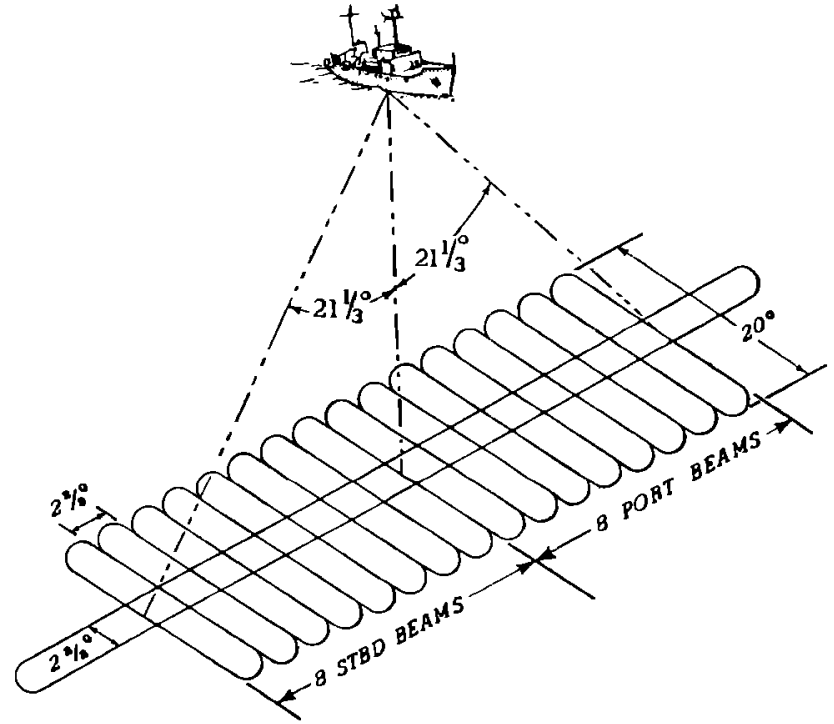

FIG. 7. Sea Beam geometry.

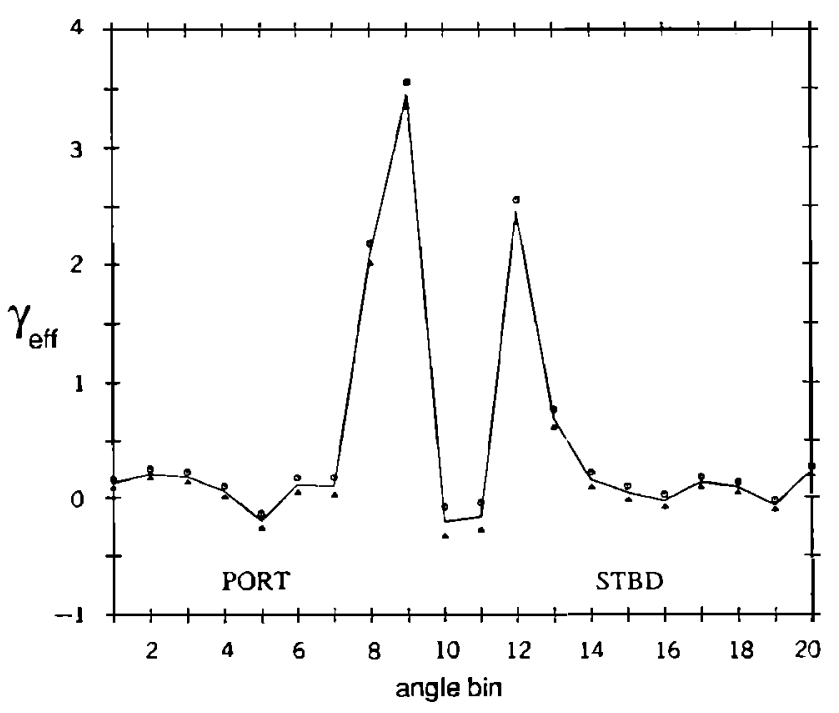

FIG. 8. Magellan Rise data: Estimated coefficient of excess $\left(\gamma_{\mathrm{ef}}\right)$ versus angle. The symbols (circles and triangles) indicate $1 \mathrm{s.d}$. about the estimate. 


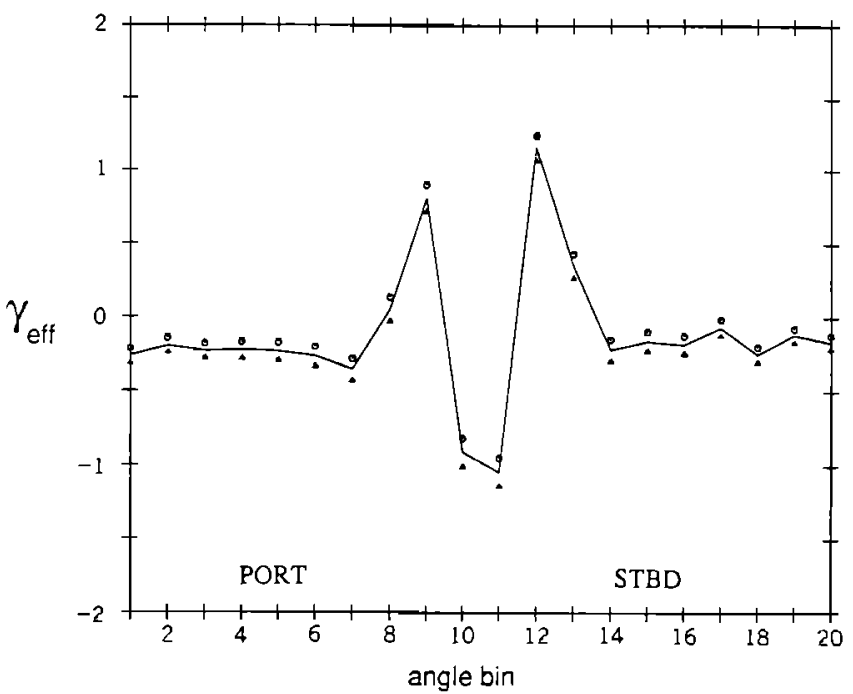

FIG. 9. Horizon Guyot data: Estimated coefficient of excess $\left(\gamma_{\text {eff }}\right)$ versus angle. The symbols (circles and triangles) indicate 1 s.d. about the estimate.

dramatically and then drops off sharply near normal incidence. This cannot be explained in terms of a restricted instantaneous insonification area because the latter actually increases in the interior beams. An alternate interpretation is possible by invoking a different scattering mechanism for near-normal incidence. As the grazing angle increases, scattering is dominated by near-specular returns from normally inclined facets of the random surface. If the total number of such contributions is relatively small, it would result in reverberation with a high $\gamma$. Finally, in the near-vertical angles, the value of $\gamma_{\text {eff }}$ falls precipitously, reaching small negative values. This abrupt change indicates the onset of the specular return, which amounts to a sizable "coherent component." One would presume that if proper normalization were possible, fitting the pdf of Eq. (23) would produce a large $Q$ value.

The Horizon Guyot data behave similarly (Fig. 9), with the exception that $\gamma_{\text {eff }}$ consistently attains negative values in the outer beams. This indicates some type of coherent component that is always present in the return, apparently in all angular bins. This explains the relatively small rise of $\gamma_{\text {eff }}$ in the interior bins and the strongly negative values in the nearvertical bins. The implication is that the underlying nonzero value of $Q$ is "holding down" $\gamma_{\text {eff }}$ preventing it from reaching the true value of $\gamma$. A number of factors may account for the presence of the "coherent component" in the Horizon Guyot set. It is conceivable that it may arise from occasional large scatterers. This is unlikely, because a large number of bottom photographs as well as visual information afforded by a number of Alvin dives indicate a uniform, sedimentcovered seafloor regime. One possibility is that the coherent
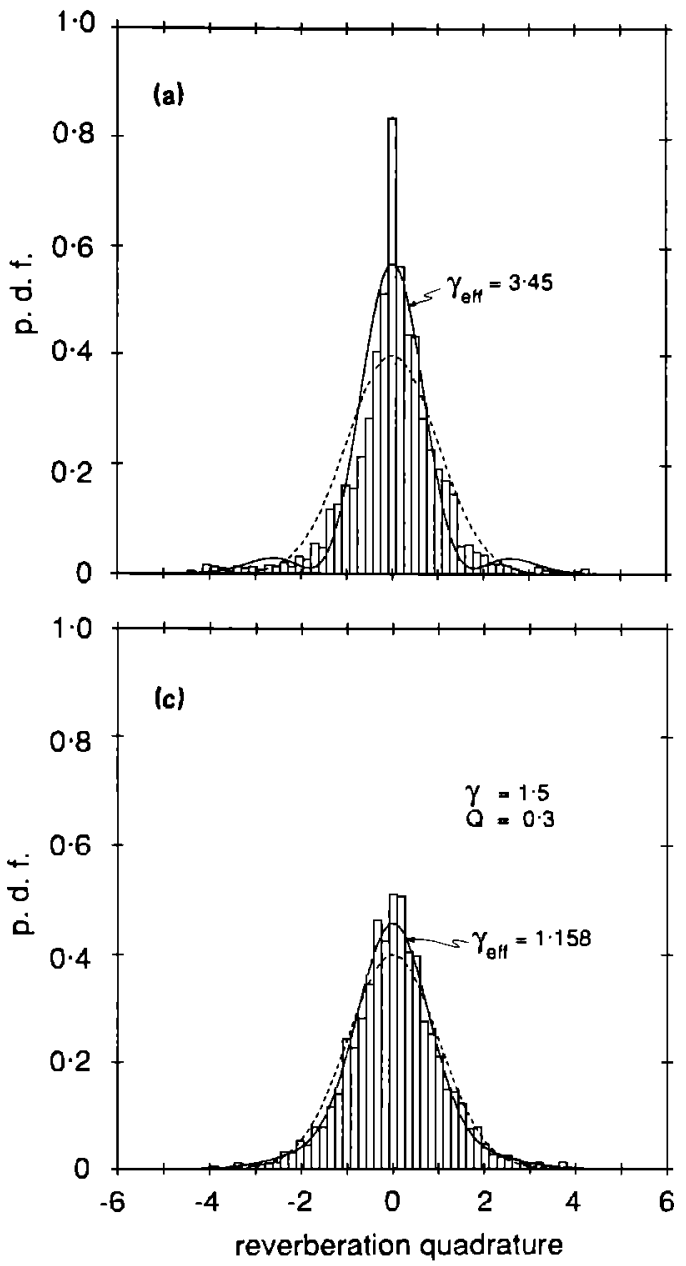

(b)

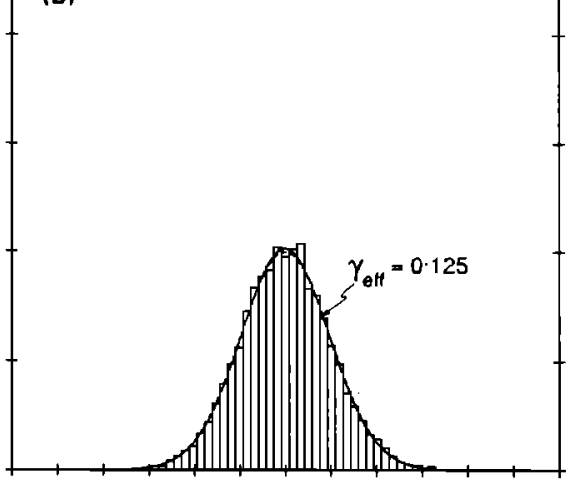

(d)

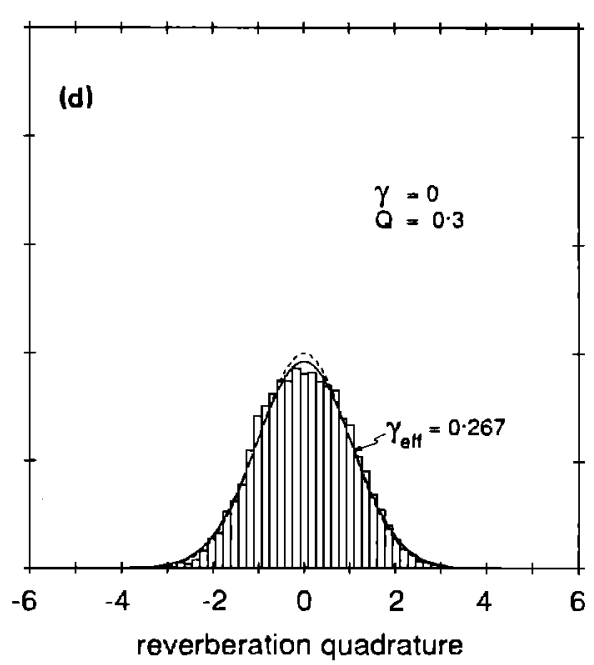

FIG. 10. Typical normalized data histograms plotted together with the nominal Gaussian pdf (dotted line) and the best fit obtained with the pdf of Eq. (23): (a) Magellan Rise, inner beams $\left(2^{\circ}-4^{\circ}\right)$, (b) Magellan Rise, outer beams $\left(18^{\circ}-20^{\circ}\right)$ (c) Horizon Guyot, inner beams $\left(2^{\circ}-4^{\circ}\right)$, and (d) Horizon Guyot, outer bins $\left(18^{\circ}-20^{\circ}\right)$. Also shown in (c) and (d) are the projected "true" values of $\gamma$ and $Q$. 
dence of volume scattering was found when analyzing these data in terms of angular dependence of seafloor backscattering strength. ${ }^{26}$ An alternative explanation is in terms of cross-talk among all the beams. In this "shallow-water" scenario, the received signal has higher power than its deepwater counterpart. The result is significantly higher levels of sidelobe and mainlobe interferences. Thus, the return in each beam is augmented by additional contributions, primarily from the neighboring beams. These contributions could have the same impact as a coherent component of the type considered here.

Representative data histograms and the best fit obtained with the pdf of Eq. (23) are shown in Fig. 10.

\section{CONCLUSIONS}

The most general pdf family supported by the pointscattering model was evaluated with computer simulations and real reverberation data. The inherent limitations of simultaneous estimation of the coefficient of excess and the "multiple glint" coherent coefficient were elucidated. The process of estimating scatterer density through $\gamma$ was demonstrated. Scatterer distributions deviating from the standard Poisson distribution were shown to lead to significantly different $\gamma$ signatures, which can potentially serve as feature vectors in the context of seafloor classification. Bottom reverberation data collected with Sea Beam were shown to reveal useful information when interpreted according to the chosen pdf model.

\section{ACKNOWLEDGMENTS}

The authors wish to thank J. Griffith for doing the artwork. This work was funded by the Office of Naval Research through Contract No. N00014-87-K-0010. Computing support was provided by the North Carolina Supercomputing Center.

' V. C. Anderson, "Frequency dependence of reverberation in the ocean," J. Acoust. Soc. Am. 41, 1467-1474 (1967).

${ }^{2}$ G. R. Wilson, "A statistical analysis of surface reverberation," J. Acoust. Soc. Am. 74, 249-255 (1983).

${ }^{3}$ G. R. Wilson and D. R. Powell, "Probability density estimates of surface and bottom reverberation," J. Acoust. Soc. Am. 73, 195-200 (1983).

${ }^{4}$ V. V. Ol'shevskii, Characteristics of Sea Reverberation (Consultants Bureau, New York, 1967).
${ }^{5}$ V. V. Ol'shevskii, Statistical Methods in Sonar (Consultants Bureau, New York, 1978).

"P. Faure, "Theoretical model of reverberation noise," J. Acoust. Soc. Am. 36, 256-259 (1964).

${ }^{7} \mathrm{D}$. Middleton, "A statistical theory of reverberation and similar first order scattered fields, part I: Waveforms and the general process," IEEE Trans. Inf. Theory IT 13, 372-392 (1967).

${ }^{8} \mathrm{D}$. Middleton, "A statistical theory of reverberation and similar first order scattered fields, part II: Moments, spectra and special distributions," IEEE Trans. Inf. Theory IT 13, 393-414 (1967).

${ }^{9} \mathrm{D}$. Middleton, "A statistical theory of reverberation and similar first order scattered fields, part III: Waveforms and fields," IEEE Trans. Inf. Theory IT 18, 35-67 (1972).

${ }^{10} \mathrm{D}$. Middleton, "A statistical theory of reverberation and similar first order scattered fields, part IV: Statistical models," IEEE Trans. Information Theory IT 18, 68-90 (1972).

"T. K. Stanton, "Sonar estimates of seafloor microroughness," J. Acoust. Soc. Am. 75, 809-818 (1984).

${ }^{12}$ R. P. Goddard, "REVGEN, High-Fidelity Simulation of Sonar Signals," Applied Physics Laboratory, University of Washington, Seattle, WA, 1985.

${ }^{13}$ R. P. Goddard, "Sonar Simulation Toolset Goals and Concepts," Applied Physics Laboratory. University of Washington, Seattle WA, 1989.

${ }^{14} \mathrm{~S}$. O. Rice, "Mathematical Analysis of Random Noise," in Selected Papers on Noise and Stochastic Processes, edited by N. Wax (Dover, New York, 1954).

${ }^{15} \mathrm{D}$. Alexandrou, "Boundary reverberation rejection via adaptive beamforming," J. Acoust. Soc. Am. 82, 1274-1290 (1987).

${ }^{16} \mathrm{D}$. Alexandrou, "Shallow Water Bathymetry: A Computer Simulation Study," in Conference Record, The global ocean, Mar. Tech. Soc. and IEEE Oceanic Eng. Soc. Seattle, WA, 1989, pp. 1208-1210.

${ }^{17} \mathrm{D}$. Alexandrou and C. de Moustier, "Adaptive noise cancelling applied to Sea Beam sidelobe interference rejection," IEEE J. Ocean. Eng. OE 13, 70-76 (1988).

${ }^{18}$ W. J. Conover, Practical Nonparametric Statistics (Wiley, New York, 1980).

${ }^{19} \mathrm{H}$. Cramer, Mathematical Methods in Statistics (Princeton U. P., Princeton, NI, 1974).

${ }^{20}$ D. Alexandrou and D. Pantzartzis, "Seafloor Classification with Neural Networks," in Conference Record, Engineering in the Ocean Environment, IEEE Oceanic Eng. Soc., Washington, DC, 1990, pp. 18-23.

${ }^{21} \mathrm{~V}$. Renard and J. P. Allenou, 'SEA BEAM multi-beam echo-sounding in "Jean Charcot." Description, evaluation and first results,' Int. Hydro. Rev. LVI, 35-67 (1979).

${ }^{22}$ H. K. Farr, "Multibeam bathymetric sonar: Sea Beam and Hydrochart," Marine Geodesy 4, 77-93 (1980).

${ }^{23}$ C. de Moustier and M. C. Kleinrock, "Bathymetric artifacts in Sea Beam data: How to recognize them, what causes them," J. Geophys. Res. 91, No B3, 3407-3424 (1986).

${ }^{24} \mathrm{C}$. de Moustier, "Beyond bathymetry: Mapping acoustic backscattering from the deep seafloor with Sea Beam," J. Acoust. Soc. Am. 79, 316-331 (1986).

${ }^{25}$ C. de Moustier and F. V. Pavlicek, "A fully transportable Sea Beam complex acoustic data acquisition system," OTC 5514, Proceedings of the Offshore Technology Conference, Houston TX, 1987, pp. 269-274.

${ }^{26} \mathrm{C}$. de Moustier and $\mathrm{D}$. Alexandrou, "Angular dependence of $12-\mathrm{kHz}$ seafloor acoustic backscatter," J. A coust. Soc. Am. 90 (1), 522-531 (1991). 